\title{
Investigating the Estimation of Primary Occupancy Patterns under Imperfect Spectrum Sensing
}

\author{
Ahmed Al-Tahmeesschi*, Miguel López-Benítez*, Janne Lehtomäki ${ }^{\dagger}$ and Kenta Umebayashi ${ }^{\ddagger}$ \\ ${ }^{*}$ Dept. of Electrical Engineering and Electronics, University of Liverpool, United Kingdom \\ ${ }^{\dagger}$ Centre for Wireless Communications, University of Oulu, Finland \\ $\ddagger$ Graduate School of Engineering, Tokyo University of Agriculture and Technology, Japan
}

\begin{abstract}
Dynamic Spectrum Access (DSA) / Cognitive Radio (CR) systems access the channel in an opportunistic, noninterfering manner with the primary network. As a result, CR performance depends on the primary channel occupancy pattern. The occupancy pattern of primary network is affected by multiple factors including time, location and frequency band. This work focuses on the time domain of spectrum sharing. The objective of this work is to study how the primary user activity pattern in the time domain (i.e., statistical distribution of the durations of idle/busy periods) affects the ability of the CR system to obtain accurate statistical information based on spectrum sensing observations. In this research, we model the primary activity pattern as a Continuous-Time Semi-Markov Chain (CTSMC). Different distributions to imitate occupancy patterns of primary network are tested by means of simulation, first when having a perfect spectrum sensing, then in the presence of imperfect spectrum sensing. It is shown that every occupancy pattern (i.e., distribution) actually leads to different levels of accuracy in the estimated statistics. A new algorithm to palliate the degrading effects of spectrum sensing errors is proposed and evaluated. The new and considered algorithms can improve the prediction of primary network statistics, however with different levels of effectiveness depending on the primary activity pattern.

Index Terms-Cognitive radio, dynamic spectrum access, spectrum sensing, spectrum awareness, primary activity statistics.
\end{abstract}

\section{INTRODUCTION}

The wireless data usage is increasing day by day due to the massive amount of applications and new trends of modern life, and it is predicted that the monthly mobile data usage will increase eight folds by 2020 compared to 2015 [1]. Multiple measurement campaigns have proven that the spectrum is underused by licensed primary users (PU) [2]. Cognitive Radio (CR) is proposed as a promising solution to solve spectrum scarcity problem. In essence, $\mathrm{CR}$ is a device that is capable of changing its transmission parameters according to surrounding primary networks [3]. In CR systems the secondary users (SU) access the PU channel in an opportunistic and non-interfering manner during PU idle times (spectrum holes) [4]. SU remain idle until a spectrum hole is detected. SU have to quickly and accurately detect spectrum holes in PU bands. However, in the case of missed detection (i.e., when SU fail to detect PU signal presence), the SU interference will last until the end of SU transmission frame [5]. A more detailed overview of CR systems can be found in [6].

CR systems can benefit from the knowledge of PU statistics to increase spectrum efficiency [7, 8], enhance PU occupancy pattern forecasts [9], select the most appropriate channel for transmission $[10,11]$ and increase the system efficiency as a result. The PU activity pattern is affected by multiple factors including for example the time when the PU channels are observed (e.g., patterns may be different during day and night times [12]), the location (indoor/outdoor and rural/urban areas), application type (e.g., voice data is modeled differently from regular internet data) [13] and sensing length [14]. This results in multiple characterisations for PU statistics of idle/busy periods. It was found in [14] that PU activity distribution varies according to sensing duration. Results have shown that the generalised Pareto distribution provides the most accurate fit for low time resolution observations (i.e., long sensing periods) while for high time resolution (i.e., short sensing periods) the best distribution fit varies depending on the PU radio technology. The PU distribution statistics can be estimated through spectrum sensing techniques. Unfortunately, spectrum sensing is imperfect resulting in an inaccurate estimation of PU statistics. Multiple methods in the literature have been proposed to increase the estimation accuracy of PU activity statistics and reduce the effect of imperfect spectrum sensing (ISS) such as [15-18], but their accuracy for different PU distributions has not been assessed (typically exponential distribution is considered to model busy/idle periods or the study is limited to a single PU distribution only). In this research, some insight is provided on how the primary activity pattern affects the ability of the CR system to obtain relevant statistical information from spectrum sensing observations under both perfect spectrum sensing (PSS) and ISS.

The rest of this work is organised as follows. First, Section II provides a formal description of different statistical distributions to model PU activity. The effects of PSS and ISS on the estimation of primary statistics are discussed in Section III. Two methods to increase the accuracy of primary statistics estimation are briefly described in Section IV. The simulation methodology is formally discussed in Section V. The obtained numerical results (through simulation) are discussed in Section VI. Finally, Section VII concludes the paper.

\section{Models for Primary USER Spectrum OcCUPANCY}

The modelling of PU occupancy depends on multiple factors including the radio technology (e.g., amateur systems, paging systems and cellular mobile communication systems) and the time scale resolution of sensing device (the duration required to perform spectrum sensing, $T_{s}$ ) as long time scales would result in different distribution patterns than short time scales, depending on the sensed radio technology. It is interesting to 
TABLE I

CONSIDERED PROBABILITY DISTRIBUTION MODELS FOR PU IDLE/BUSY PERIOD DURATIONS. DISTRIBUTION NAMES: E (EXPONENTIAL), GE

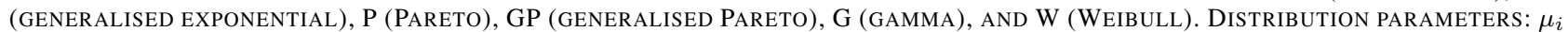
(LOCATION), $\lambda_{i}$ (SCALE), AND $\alpha_{i}$ (SHAPE). $T_{i}$ REPRESENTS THE PERIOD LENGTH. $\mathbb{E}\{\cdot\}$ AND $\mathbb{V}\{\cdot\}$ REPRESENT THE MEAN AND THE VARIANCE OF THE DISTRIBUTION, RESPECTIVELY. $\psi(\cdot)$ IS THE DIGAMMA FUNCTION $[19,6.3 .1]$ AND $\psi^{\prime}(\cdot)$ IS ITS DERIVATIVE. $\gamma(\cdot, \cdot)$ IS THE LOWER INCOMPLETE GAMMA FUNCTION [19,6.5.2] AND $\Gamma(\cdot)$ IS THE (COMPLETE) GAMMA FUNCTION [19,6.1.1]. (REPRODUCED FROM [14]).

\begin{tabular}{|c|c|c|}
\hline Distribution function & Parameters & Moments \\
\hline$F_{E}\left(T_{i} ; \mu_{i}, \lambda_{i}\right)=1-e^{-\lambda_{i}\left(T_{i}-\mu_{i}\right)}$ & $\begin{array}{l}T_{i} \geq \mu_{i}>0 \\
\quad \lambda_{i}>0\end{array}$ & $\begin{array}{l}\mathbb{E}\left\{T_{i}\right\}=\mu_{i}+\frac{1}{\lambda_{i}} \\
\mathbb{V}\left\{T_{i}\right\}=\frac{1}{\lambda_{i}^{2}}\end{array}$ \\
\hline$F_{G E}\left(T_{i} ; \mu_{i}, \lambda_{i}, \alpha_{i}\right)=\left[1-e^{-\lambda_{i}\left(T_{i}-\mu_{i}\right)}\right]^{\alpha_{i}}$ & $\begin{array}{c}T_{i} \geq \mu_{i}>0 \\
\lambda_{i}>0 \\
\alpha_{i}>0\end{array}$ & $\begin{array}{l}\mathbb{E}\left\{T_{i}\right\}=\mu_{i}+\frac{\psi\left(\alpha_{i}+1\right)-\psi(1)}{\lambda_{i}} \\
\mathbb{V}\left\{T_{i}\right\}=\frac{\psi^{\prime}(1)-\psi^{\prime}\left(\alpha_{i}+1\right)}{\lambda_{i}^{2}}\end{array}$ \\
\hline$F_{P}\left(T_{i} ; \lambda_{i}, \alpha_{i}\right)=1-\left(\frac{\lambda_{i}}{T_{i}}\right)^{\alpha_{i}}$ & $\begin{array}{c}T_{i} \geq \lambda_{i} \\
\lambda_{i}>0 \\
\alpha_{i}>2\end{array}$ & $\begin{aligned} \mathbb{E}\left\{T_{i}\right\} & =\frac{\alpha_{i} \lambda_{i}}{\alpha_{i}-1} \\
\mathbb{V}\left\{T_{i}\right\} & =\frac{\alpha_{i} \lambda_{i}^{2}}{\left(\alpha_{i}-1\right)^{2}\left(\alpha_{i}-2\right)}\end{aligned}$ \\
\hline$F_{G P}\left(T_{i} ; \mu_{i}, \lambda_{i}, \alpha_{i}\right)=1-\left[1+\frac{\alpha_{i}\left(T_{i}-\mu_{i}\right)}{\lambda_{i}}\right]^{-1 / \alpha_{i}}$ & $\begin{array}{c}T_{i} \geq \mu_{i}\left(\alpha_{i} \geq 0\right) \\
T_{i} \in\left[\mu_{i}, \mu_{i}-\frac{\lambda_{i}}{\alpha_{i}}\right]\left(\alpha_{i}<0\right) \\
\mu_{i}, \lambda_{i}>0, \alpha_{i}<1 / 2\end{array}$ & $\begin{aligned} \mathbb{E}\left\{T_{i}\right\} & =\mu_{i}+\frac{\lambda_{i}}{1-\alpha_{i}} \\
\mathbb{V}\left\{T_{i}\right\} & =\frac{\lambda_{i}^{2}}{\left(1-\alpha_{i}\right)^{2}\left(1-2 \alpha_{i}\right)}\end{aligned}$ \\
\hline$F_{G}\left(T_{i} ; \mu_{i}, \lambda_{i}, \alpha_{i}\right)=\frac{\gamma\left(\alpha_{i}, \frac{T_{i}-\mu_{i}}{\lambda_{i}}\right)}{\Gamma\left(\alpha_{i}\right)}$ & $\begin{array}{c}T_{i} \geq \mu_{i}>0 \\
\lambda_{i}>0 \\
\alpha_{i}>0 \\
\end{array}$ & $\begin{array}{l}\mathbb{E}\left\{T_{i}\right\}=\mu_{i}+\lambda_{i} \alpha_{i} \\
\mathbb{V}\left\{T_{i}\right\}=\lambda_{i}^{2} \alpha_{i}\end{array}$ \\
\hline$F_{W}\left(T_{i} ; \mu_{i}, \lambda_{i}, \alpha_{i}\right)=1-\exp \left[-\left(\frac{T_{i}-\mu_{i}}{\lambda_{i}}\right)^{\alpha_{i}}\right]$ & $\begin{array}{l}T_{i} \geq \mu_{i}>0 \\
\lambda_{i}>0 \\
\alpha_{i}>0\end{array}$ & $\begin{array}{l}\mathbb{E}\left\{T_{i}\right\}=\mu_{i}+\lambda_{i} \Gamma\left(1+\frac{1}{\alpha_{i}}\right) \\
\mathbb{V}\left\{T_{i}\right\}=\lambda_{i}^{2}\left[\Gamma\left(1+\frac{2}{\alpha_{i}}\right)-\Gamma^{2}\left(1+\frac{1}{\alpha_{i}}\right)\right]\end{array}$ \\
\hline
\end{tabular}

note that most researches assume an exponential distribution to model the binary PU holding states (idle/busy) for both large/small $T_{s}$. This was proven to be inaccurate [14], as for long time scale the generalised Pareto distribution provides the best fit for nearly all the studied bands and radio technologies, while for short time scales the best distribution fit depends mainly on the radio technology type. Most of the previous works focused on exponential distribution or more realistic but complex distributions. The novelty of this work comes from the fact that no previous works have considered analyses of multiple PU distributions. In this research, it is assumed that the PU occupancy pattern is in the time dimension and can be modelled with a Continuous-Time Semi-Makarov Chain (CTSMC) [2] as the holding distribution for busy/idle periods can follow any arbitrary distribution. The considered distributions are the same as in [14]: exponential [20], generalised exponential [21], Pareto [22], generalised Pareto [23], gamma [20] and Weibull [20]. Table I shows the cumulative distribution function (CDF) for the considered models of PU binary occupancy patterns.

\section{ESTIMATION OF PU STATISTICS UNDER ISS}

CR performs spectrum sensing at a finite rate and duration $T_{s}$ in order to provide a binary decision of the channel state, namely idle $\left(H_{0}\right)$ or busy $\left(H_{1}\right)$, as it can be seen in Fig. 1 . Period durations $\widehat{T}_{i}$ (where $i=0$ denotes idle period and $i=1$ denotes busy period) can be calculated from spectrum sensing decisions as shown in Fig. 1(a). When CR operates under high PU transmission power, PSS can be assumed. Unfortunately, in most cases CR operates under low PU transmission power. In this case sensing errors will arise and ISS occurs. The main error types are false alarms (the detector announces the PU channel as busy when it is actually idle) and missed detections (the detector announces the channel idle in the presence of
PU signal). These errors, which are characterised by their probabilities $P_{f a}$ and $P_{m d}$ respectively, lead to incorrect estimation of periods durations $\breve{T}_{i}$ as shown in Fig. 1(b). The probability of occurrence of sensing errors depends on the employed sensing method (e.g., energy detection, matched filter detection, cyclostationary/feature-based detection, etc.) and approach (e.g., single channel sensing, multi-channel FFTbased sensing with different time/frequency resolutions, etc.). The impact of using different sensing methods and approaches is captured by the corresponding $P_{f a}$ and $P_{m d}$ probabilities. Therefore, this study is valid regardless of the particular sensing method/approach considered by the CR system. A detailed investigation on sensing errors can be found in [18].

In this work, the main aim is to investigate the impact of different PU occupancy models under both PSS and ISS, and determine whether algorithms that have been designed and evaluated for a particular PU distribution can still achieve a high accuracy in statistics prediction when the PU pattern follows a different distribution.

\section{Methods to Palliate the efFects of ISS}

PU statistics can be estimated directly from the period durations $\breve{T}_{i}$ observed under ISS. However, since these observed periods are affected by sensing errors, the resulting estimated statics will be inaccurate as well. In order to improve the accuracy of the estimated statistics, some methods have been proposed to attempt to reconstruct the period durations $\widehat{T}_{i}$ that would be observed under PSS. Such methods usually rely on some a priori knowledge of the PU activity pattern. This work considers two methods that rely on the knowledge of the minimum PU activity time, denoted as $\mu_{i}\left(0<T_{s}<\mu_{i}\right)$. There are multiple methods to obtain the minimum activity duration, including regional beacon signals with real-time information about minimum activity time for PU present in 


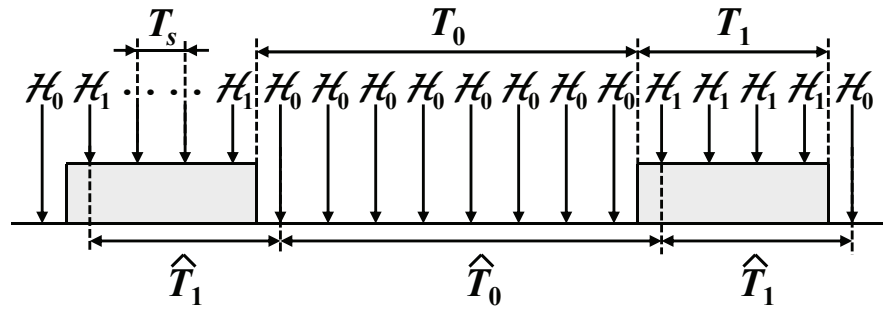

(a)

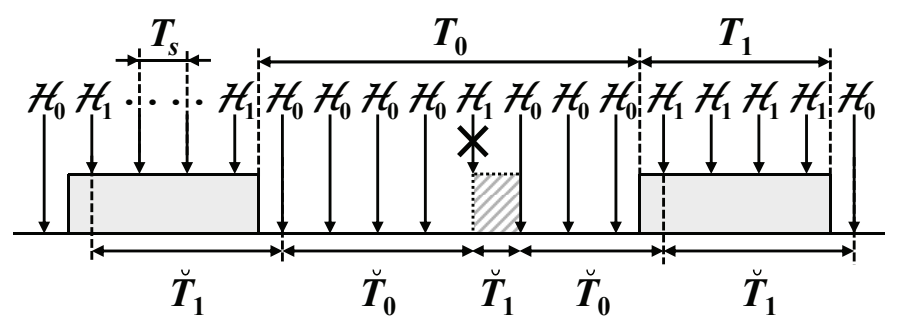

(b)

Fig. 1. Estimation of period durations from spectrum sensing decisions: (a) under perfect spectrum sensing, (b) under imperfect spectrum sensing [18] $\widehat{T}_{i}$ represents the estimated period under perfect spectrum sensing and $\breve{T}_{i}$ represents the estimated period under imperfect spectrum sensing.

this geographical area [24], or by utilizing blind estimation methods at SU [25] to determine the minimum PU activity time.

\section{A. Method 1}

In this method all the observed periods $\breve{T}_{i, n}$ (where $n \in \mathbb{N}^{+}$ denotes the index within the sequence of observed periods) are checked and every period with duration shorter than the PU minimum activity time $\left(\breve{T}_{i, n}<\mu_{i}\right)$ is discarded [18].

\section{B. Method 2}

In most cases sensing errors occur in individual isolated sensing events. This can be explained by the fact that the sensing period $T_{s}$ can be assumed to be much longer than the channel coherence time in most practical scenarios (i.e., missed detections are not typically correlated) and the internal thermal noise of a receiver is an uncorrelated random process (i.e., false alarms are uncorrelated as well). However, as can be observed in Fig. 2 that in some cases (in particular when the probability of sensing errors is higher) sometimes bursts of two or more consecutive erroneous sensing events may occur as well. This method is designed to detect the occurrence of both isolated errors and bursts of sensing errors and then attempt to reconstruct the periods $\widehat{T}_{i}$ that would be observed under PSS.

First, a threshold $\beta T_{s}<\mu_{i}$ (with $\beta \in \mathbb{N}^{+}$) is defined, which can be tuned as explained in section VI. Starting from an initial estimated period $\breve{T}_{i, n}$ the durations of all subsequent periods (both busy and idle) are checked until a period of the opposite type with a duration greater than $\beta T_{s}$ (i.e., $\left.\breve{T}_{1-i, n+N}>\beta T_{s}\right)$ is found. A reconstructed period of the same type (idle/busy) as $\breve{T}_{i, n}$ is then estimated by adding the durations $\breve{T}_{i, n}+\ldots \breve{T}_{i, n+N-1}$. The period $\breve{T}_{1-i, n+N}$ (which is of a different type than the previously reconstructed period) is then taken as the starting point for a new reconstruction

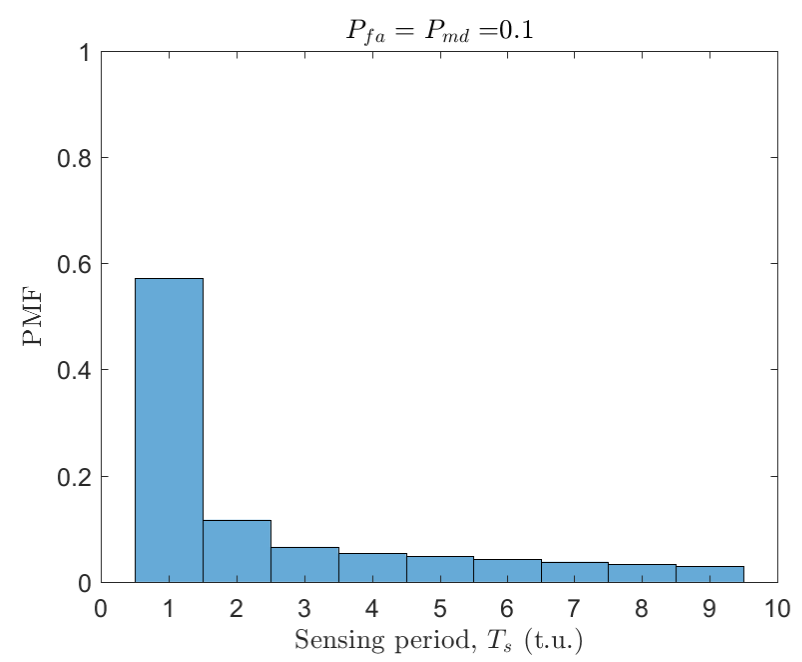

Fig. 2. The probability mass function (PMF) of the number of consecutive sensing events $\left(T_{i}\right)$ affected by sensing errors (obtained from simulations).

based on the same principle. This process is repeated over the sequence of estimated periods so that all periods shorter than $\beta T_{s}$ (which can be assumed to be short periods resulting from sensing errors) will be added in an attempt to reconstruct the sequence of busy/idle periods $\widehat{T}_{i}$. After applying the method above, some of the reconstructed periods might be shorter than $\mu_{i}$, thus indicating the presence of a few incorrectly reconstructed periods. Therefore a second step is performed after the reconstruction process above where all the reconstructed periods shorter than $\mu_{i}$ are discarded and not included in the computation of the PU activity statistics.

\section{Simulation methodology}

The performance of the statistical distributions considered in this work is evaluated by using MATLAB computer simulations. The generation of activity periods follows similar steps as proposed in [18]:

1) Generate idle and busy periods' lengths $T_{i}$, both following one of the distributions shown in Table I.

2) Perform idle/busy sensing decisions $H_{0} / H_{1}$ on the generated sequence in step 1 every $T_{s}$ time units (t.u.).

3) Add independent random errors (with $P_{f a}>0$ and $\left.P_{m d}>0\right)$ in the sequence resulting from step 2 .

4) Using the new $H_{0} / H_{1}$ sequence from step 3 , calculate the period lengths $\breve{T}_{i}$ that would be estimated under ISS.

5) Process the sequence of period lengths resulting from step 4 in order to reconstruct the original periods by making use of one of the two methods described in Section IV.

6) Compute the CDF of the idle/busy periods obtained in steps $4 \& 5$ and compare with the CDF of the original durations in step 1 .

The comparison between the estimated and the original distributions is performed using the well-known KolmogorovSmirnov (KS) distance [26], defined as:

$$
D_{K S}=\sup _{T_{i}}\left|F_{T_{i}}\left(T_{i}\right)-F_{\breve{T}_{i}}\left(T_{i}\right)\right|
$$


TABLE II

DISTRIBUTION PARAMETERS.

\begin{tabular}{|c|c|c|}
\hline Distribution & Shape $\left(\alpha_{i}\right)$ & Scale $\left(\lambda_{i}\right)$ \\
\hline E & - & 0.25 \\
\hline GE & 29.179 & 0.0993 \\
\hline P & 2.001 & 25.355 \\
\hline GP & 0.25 & 30 \\
\hline G & 9.499 & 4.215 \\
\hline W & 3.167 & 44.7721 \\
\hline
\end{tabular}

Note that the generated sequence of idle/busy lengths $T_{i}$ in step 1 contains real positive values and its $\mathrm{CDF} F_{T_{i}}\left(T_{i}\right)$ has a continuous domain, while the sequences resulting from steps 4 and 5 contain values $\breve{T}_{i}$ that are integer multiples of the sensing period $\left(\breve{T}_{i}=k T_{s}, k \in \mathbb{N}^{+}\right)$and their CDFs $F_{\breve{T}_{i}}\left(\breve{T}_{i}\right)$ have a discrete domain. Since it is not possible to compare continuous and discrete sets, the discrete set $F_{\breve{T}_{i}}\left(\breve{T}_{i}\right)$ is interpolated to produce $F_{\breve{T}_{i}}\left(T_{i}\right)$, which is compared in (1) with the distribution of the original lengths $F_{T_{i}}\left(T_{i}\right)$.

\section{NUMERICAL RESULTS}

The study is performed considering the distributions shown in Table I (exponential, generalised exponential, Pareto, generalised Pareto, gamma and Weibull). In order to maintain a fair comparison between the different distributions, their parameters $\left(\lambda_{i}\right.$ and $\left.\alpha_{i}\right)$ are configured as shown in Table II to provide the same minimum activity time $\left(\mu_{i}=10\right.$ t.u. $)$ and the same mean period duration $\left(\mathbb{E}\left\{T_{i}\right\}=50\right.$ t.u.). The same parameters are considered for both busy and idle periods, which leads to a channel duty cycle (DC) of $50 \%(\Psi=0.5)$.

To select the best threshold for method 2, multiple threshold values have been tested as can be seen in Fig. 3. In this simulation, the idle/busy periods followed a generalized Pareto distribution. The best results (smallest KS distance) are obtained for $\beta=3$ (Fig. 3). This threshold will be used for the following simulation results.

Fig 4 shows the CDFs of the considered distributions for original generated periods $\left(T_{i}\right)$. As it can be appreciated, some distributions show similar trends. In particular, three different groups of distributions can be distinguished. The first group is generalised Pareto and exponential distributions, the second group contains gamma, generalised exponential and Weibull distributions, while the last group contains only the Pareto distribution which behaves differently from other distributions. This observation will serve to explain later on the differences and similarities observed in the obtained results.

Fig. 5 shows the performance of PSS in comparison with the original periods. In this case, the only source of error is the use of a finite sensing period $T_{s}$, which results in all the observed periods being integer multiples of $T_{s}$ (i.e., $\widehat{T}_{i}=k T_{s}, k \in$ $\mathbb{N}^{+}$). As appreciated, the estimation error can be decreased by reducing $T_{s}$ (i.e., increasing the time resolution improves the accuracy). Moreover it can also be noted that there are some differences in the accuracy obtained for different distributions, however these are mainly minor differences, except for the Pareto distribution, which shows a slightly different trend as $T_{s}$

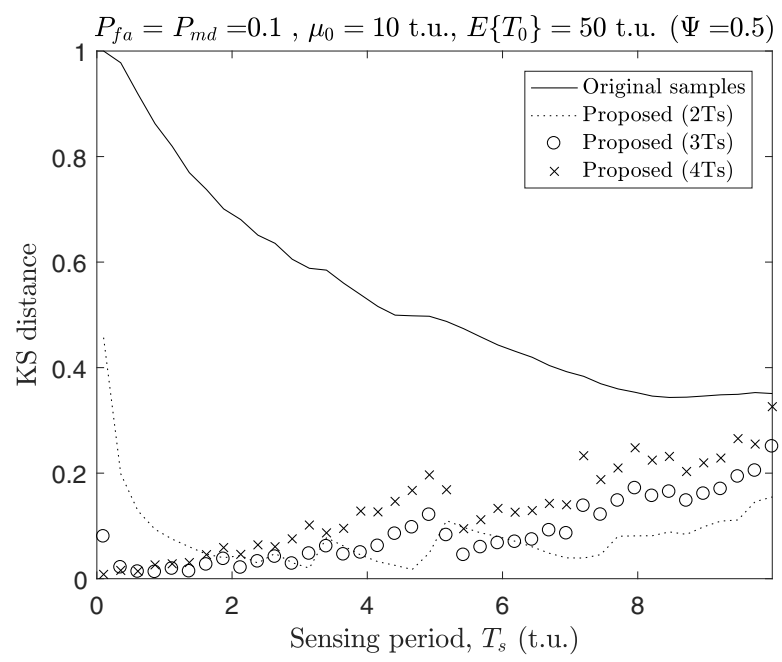

Fig. 3. Performance of method proposed in this work.

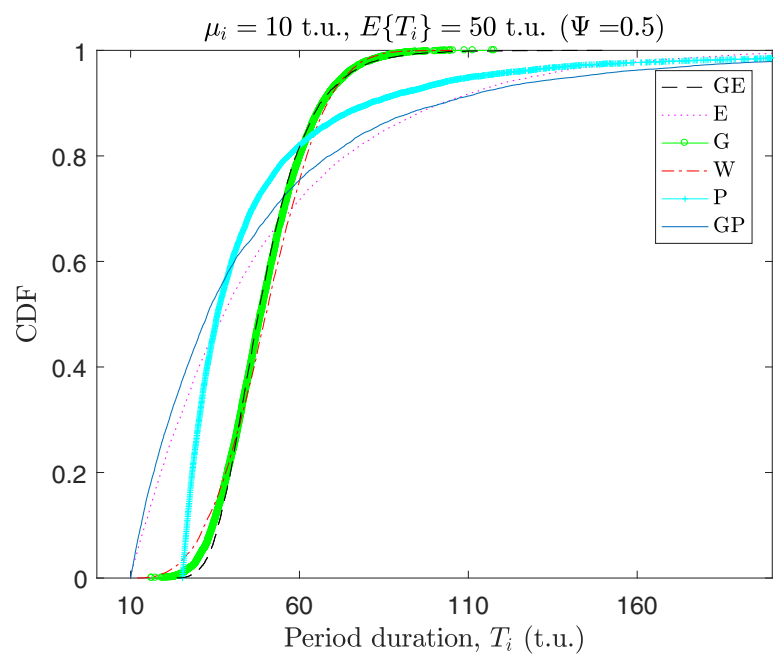

Fig. 4. CDF of idle/busy periods ( $\mu_{i}=10$ t.u., $E\left\{T_{i}\right\}=50$ t.u., $\Psi=0.5$ ).

increases (note in Fig. 4 that the CDF of the Pareto distribution behaves differently from all other distributions).

Fig. 6 shows the estimation accuracy for the CDF of the PU busy/idle periods under ISS in terms of the KS distance (with respect to the original periods $T_{i}$ obtained from of step 1) as a function of the sensing period. The results are shown for the following cases: no reconstruction methods are used and the PU statistics are estimated from the periods $\breve{T}_{i}$ observed under ISS (left), the periods $\breve{T}_{i}$ are processed according to reconstruction method 1 (centre), and the periods $\breve{T}_{i}$ are processed according to reconstruction method 2 (right). The top row shows the results obtained for $P_{f a}=P_{m d}=0.01$ while the bottom row show the results obtained for $P_{f a}=$ $P_{m d}=0.1$. Simulations are performed for $\Psi=0.5$ (medium load). Nevertheless, other duty cycles ( $\Psi=0.2$ and $\Psi=0.8$ ) were analysed as part of this work and, even though they are not shown due to the lack of space, they showed similar trends.

As it can be appreciated from Fig. 6, the accuracy is 


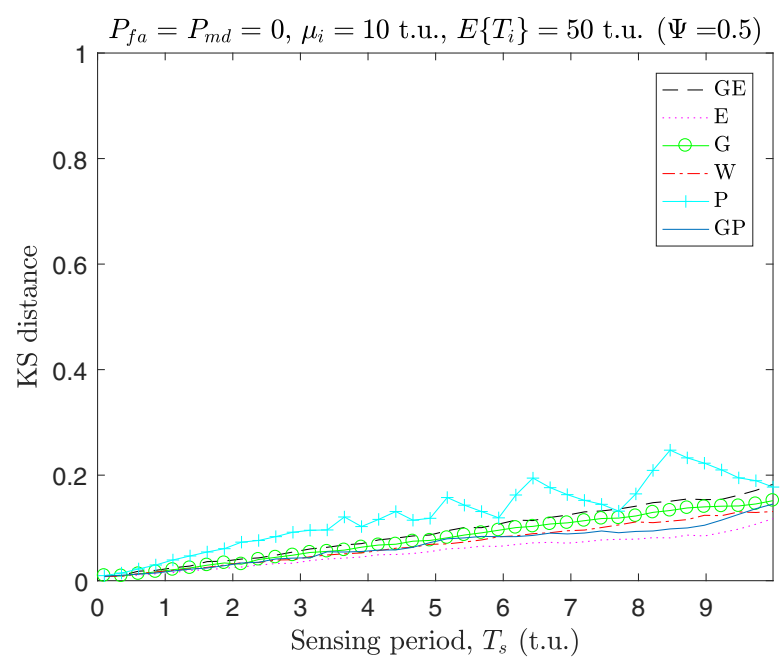

Fig. 5. Estimation accuracy for distributions under PSS.

in general better when having a lower probability of error (top row). Nevertheless, the considered reconstruction methods can provide better accuracy compared to the case where no reconstruction is considered (i.e., when the PU statistics are computed straight from the periods observed under ISS). As opposed to the PSS case shown in Fig. 5, the accuracy under ISS degrades as the sensing period $T_{s}$ decreases. This is due to the fact that reducing $T_{s}$ increases the number of sensing events per PU idle/busy period and therefore the probability of having sensing errors within each of the PU idle/busy periods.

For the case where no reconstruction methods are applied, the accuracy of the estimated PU statistics depends on the considered distributions. Notice that similar distributions lead to similar levels of accuracy. Both generalised Pareto and exponential distributions, which are similar as shown in Fig. 4, lead to the highest accuracy and immunity against sensing errors. On the other hand, the generalised exponential, gamma and Weibull distributions, which are also similar among them, provide relatively similar estimation accuracies, while the Pareto distribution results in the lowest accuracy (this observation holds for both cases of sensing errors, i.e., low $P_{f a}=P_{m d}=0.01$ and high $P_{f a}=P_{m d}=0.1$ probabilities).

For method 1, similar comments as above can be made. However, in this case some improvement in the accuracy of the estimated PU statistics is obtained for all the distributions as a result of the reconstruction operation performed by this method. It is interesting to note that for the case of low probability of sensing errors $\left(P_{f a}=P_{m d}=0.01\right)$ there are some cases where the accuracy of the estimated statistics is nearly perfect $\left(D_{K S} \approx 0\right)$. As shown in Fig. 6, this occurs for the generalised Pareto and exponential distributions when the sensing period is close to the minimum PU activity time $\left(T_{s} \approx \mu_{i}\right)$ but not for any other distributions. This may incorrectly lead to the conclusion that certain methods can provide a nearly perfect estimation of the PU statistics when this actually depends on the particular PU distribution considered (i.e., it may be true for some PU systems but not for some others and the efficacy of a certain method may actually depend on the PU system over which it is applied).

For method 2, the threshold is set to $\beta=3$ as mentioned. Note that this method is more complex than method 1 and the level of improvement is notably higher. Notice that in this case there is a wide range of (low) values of the sensing period $T_{s}$ that can provide a nearly perfect estimation $\left(D_{K S} \approx 0\right)$. When the probability of sensing errors is low $\left(P_{f a}=P_{m d}=0.01\right)$ this observation holds for all the distributions (even though for the Pareto distribution the range is slightly shorter). However for higher probability of sensing errors $\left(P_{f a}=P_{m d}=0.1\right)$ this observation only holds for the generalised Pareto and exponential distributions, where both can achieve nearly perfect accuracy over a wide range (for other distributions there is a lower bound below which the error cannot decrease). In contrast with method 1 , the results for method 2 are not so sensitive to the particular PU occupancy pattern (distribution).

Finally, as it can be appreciated from Fig. 6, the efficiency of the considered methods may depend to different extents on the PU activity statistics (i.e., some methods may be more effective for certain PU distributions than for others while some other methods may be less sensitive to the particular PU distribution). If this is not adequately considered then the performance of a real system design could be under or overestimated, thus leading to unexpected behaviours/performances. This important observation should be carefully taken into account in future studies on DSA/CR systems, where traditionally only one distribution model is considered for the PU idle/busy period durations (typically the exponential distribution, which has been shown to be inaccurate as discussed in Section II, or a more accurate model but without considering other possible PU activity patterns as well).

\section{CONCLUSION}

While multiple models have been proposed in the literature to model PU occupancy patterns, most of the existing studies are based on one single model (typically exponentially distributed idle/busy periods). In this work multiple models to imitate PU occupancy pattern have been considered and their impact on the accuracy of methods to estimate the PU activity statistics has been investigated by means of simulations under both PSS and ISS. The obtained results have demonstrated that the efficiency of different methods may depend on the particular PU activity statistics. As a matter of fact, some methods may be less sensitive than others to the particular PU distribution and exhibit more similar or dissimilar levels of accuracy under different operating conditions. Future studies on DSA/CR systems in general, and on methods for the estimation of PU activity statistics in particular, should not be constrained to a single model for the distribution of PU idle/busy periods (as it has usually been the case in the past) but consider a sufficiently broad range of models for a more accurate and comprehensive performance evaluation.

\section{REFERENCES}

[1] Cisco, "Cisco visual networking index: Global mobile data traffic forecast update, 2015-2020," Feb 2016, White Paper. 

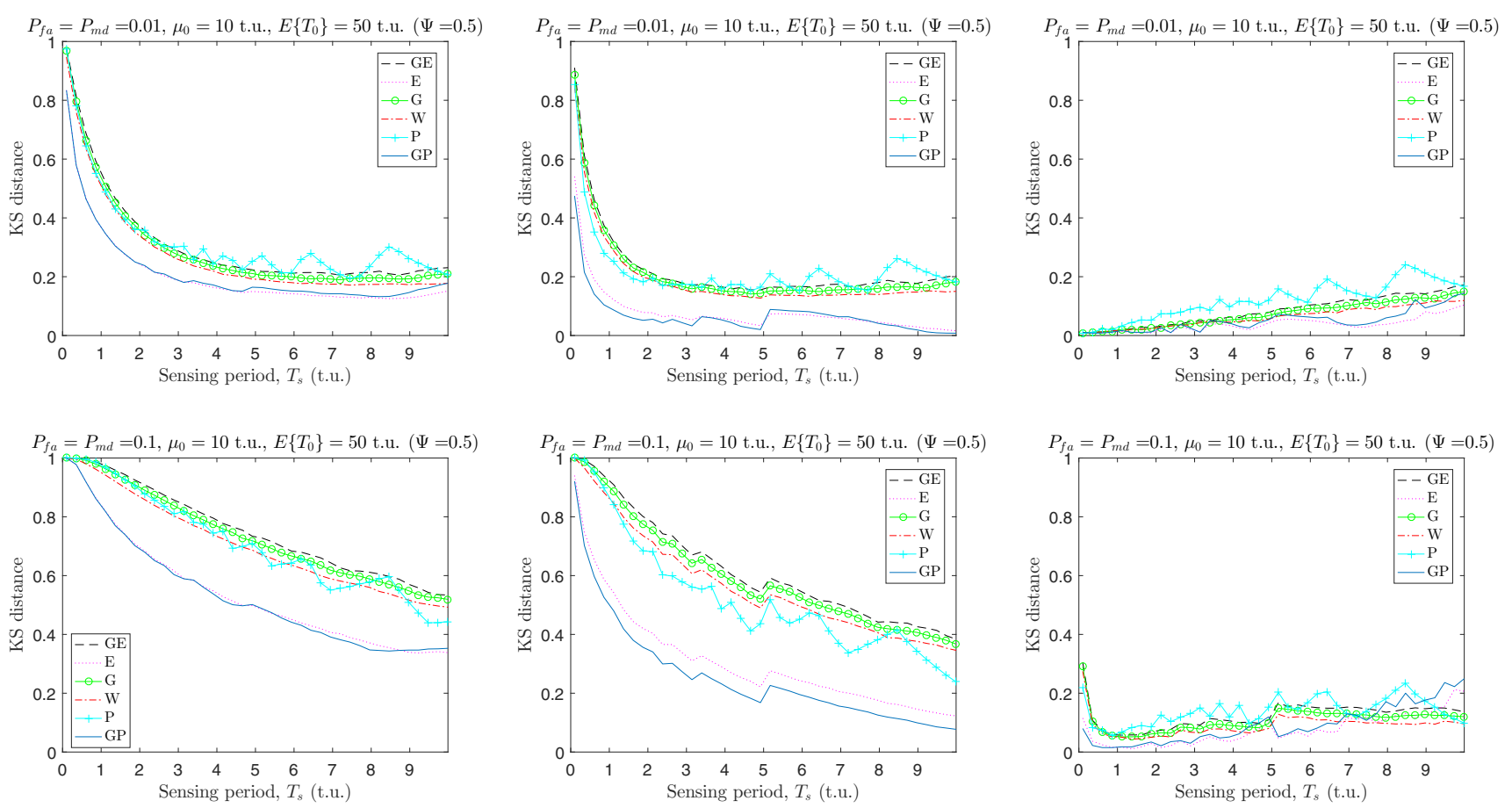

Fig. 6. Estimation accuracy for distributions under ISS (details in the text).

[2] M. López-Benítez and F. Casadevall, "Spectrum Occupancy in Realistic Scenarios and Duty Cycle Model for Cognitive Radio," Adv. Electron.Telecommun., vol. 1, no. Apr, pp. 26-34, 2010.

[3] S. Haykin, "Cognitive radio: Brain-empowered wireless communications," IEEE J. Sel. Areas Comms., vol. 23, no. 2, pp. 201-220, Feb. 2005.

[4] M. López-Benítez and F. Casadevall, Spectrum Usage Models for the Analysis, Design and Simulation of Cognitive Radio Networks. Dordrecht: Springer Netherlands, 2012, pp. 27-73. [Online]. Available: http://dx.doi.org/10.1007/978-94-007-1827-2_2

[5] K. J. R. Liu and B. Wang, Cognitive Radio Networking and Security: A Game-Theoretic View, 1st ed. New York, NY, USA: Cambridge University Press, 2010.

[6] Y. C. Liang, K. C. Chen, G. Y. Li, and P. Mahonen, "Cognitive radio networking and communications: an overview," IEEE Transactions on Vehicular Technology, vol. 60, no. 7, pp. 3386-3407, Sept 2011.

[7] K. Umebayashi, K. Moriwaki, R. Mizuchi, H. Iwata, S. Tiiro, J. Lehtomäki, M. López-Benítez, and Y. Suzuki, "Simple primary user signal area estimation for spectrum measurement," IEICE Transactions on Communications, vol. E99-B, no. 2, pp. 523-532, Feb. 2016

[8] E. Jung and X. Liu, "Opportunistic spectrum access in multiple-primaryuser environments under the packet collision constraint," IEEE/ACM Trans. Networking, vol. 20, no. 2, pp. 501-514, Apr. 2012.

[9] V. K. Tumuluru, P. Wang, and D. Niyato, "A neural network based spectrum prediction scheme for cognitive radio," in Proc. 2010 IEEE Int'l. Conf. Comms. (ICC 2010), May 2010, pp. 1-5.

[10] X. Liu, B. Krishnamachari, and H. Liu, "Channel selection in multichannel opportunistic spectrum access networks with perfect sensing," in Proc. 2010 IEEE Int'l. Symp. Dyn. Spect. Access Networks (DySPAN 2010), Apr. 2010, pp. 1-8.

[11] J. Vartiainen, M. Höyhtyä, J. Lehtomäki, and T. Bräysy, "Priority channel selection based on detection history database," in Proc. Fifth Int'l. Conf. Cognitive Radio Oriented Wireless Networks \& Comms. (CROWNCOM 2010), Jun. 2010, pp. 1-5.

[12] M. López-Benítez and F. Casadevall, "Empirical time-dimension model of spectrum use based on a discrete-time markov chain with deterministic and stochastic duty cycle models," IEEE Transactions on Vehicular Technology, vol. 60, no. 6, pp. 2519-2533, July 2011.

[13] K. Sithamparanathan and A. Giorgetti, Cognitive radio techniques. Artech House, 2012.

[14] M. López-Benítez and F. Casadevall, "Time-dimension models of spec- trum usage for the analysis, design, and simulation of cognitive radio networks," IEEE Transactions on Vehicular Technology, vol. 62, no. 5, pp. 2091-2104, Jun 2013.

[15] W. Gabran, C. H. Liu, P. Pawelczak, and D. Cabric, "Primary user traffic estimation for dynamic spectrum access," IEEE Journal on Selected Areas in Communications, vol. 31, no. 3, pp. 544-558, March 2013.

[16] C. H. Liu, P. Pawelczak, and D. Cabric, "Primary user traffic classification in dynamic spectrum access networks," IEEE Journal on Selected Areas in Communications, vol. 32, no. 11, pp. 2237-2251, November 2014.

[17] J. Lehtomäki, M. López-Benítez, K. Umebayashi, and M. Juntti, "Improved channel occupancy rate estimation," IEEE Transactions on Communications, vol. 63, no. 3, pp. 643-654, March 2015.

[18] M. López-Benítez, "Can primary activity statistics in cognitive radio be estimated under imperfect spectrum sensing?" in 2013 IEEE 24th Annual International Symposium on Personal, Indoor, and Mobile Radio Communications (PIMRC), Sept 2013, pp. 750-755.

[19] M. Abramowitz and I. A. Stegun, Handbook of mathematical functions with formulas, graphs, and mathematical tables, 10th ed. New York: Dover, 1972.

[20] J. F. Lawless, Statistical models and methods for lifetime data. Wiley, 1982.

[21] R. D. Gupta and D. Kundu, "Generalized exponential distributions," Australian and New Zealand Journal of Statistics, vol. 41, no. 2, pp. 173-188, Jun. 1999.

[22] D. C. Montgomery and G. C. Runger, Applied statistics and probability for engineers, 3rd ed. John Wiley \& Sons, 2003.

[23] T. Öztekin, "Comparison of parameter estimation methods for the threeparameter generalized Pareto distribution," Turkish Journal of Agriculture and Forestry, vol. 29, no. 6, pp. 419-428, Dec. 2005.

[24] M. López-Benítez, "Cognitive radio," in Heterogeneous cellular networks: Theory, simulation and deployment. Cambridge University Press, 2013, ch. 13.

[25] M. López-Benítez, F. Casadevall, A. Umbert, J. Perez-Romero, R. Hachemani, J. Palicot, and C. Moy, "Spectral occupation measurements and blind standard recognition sensor for cognitive radio networks," in 2009 4th International Conference on Cognitive Radio Oriented Wireless Networks and Communications, June 2009, pp. 1-9.

[26] W. H. Press, S. A. Teukolsky, W. T. Vetterling, and B. P. Flannery, Numerical recipes: The art of scientific computing, 3rd ed. Cambridge University Press, 2007. 